\title{
Ein aufwändiger Weg zur Diagnose: Fallbericht eines Granularzelltumors der Brust
}

\author{
P. Polzer, S. Weigel
}

\section{Fallbericht}

Wir berichten über eine 50 -jährige beschwerdefreie prämenopausale Frau, bei der im Rahmen der Erstteilnahme am Mammografie-Screeningprogramm rechts axillär ein irreguläres Verdichtungsareal auffiel. In den ergänzend durchgeführten Zielkompressionsaufnahmen wurde das Verdichtungsareal als unscharf abgrenzbarer bis gering spikulierter Herd bestätigt ( $\triangleright$ Abb. 1). Unter Kenntnis der Mammografie war sonografisch ein echoarmer, spikulierter Herd mit zulaufender Gefäßstruktur darstellbar ( $\triangleright$ Abb. 2) und es wurde die Indikation zur ultraschallgestützten Stanzbiopsie (14 G) gestellt. Die Histologie der vier bildgebend repräsentativen Stanzzylinder ergab eine Kombination aus Fettgewebe, Fibroseareal sowie vereinzelten histiozytenartigen Zellen (B2), womit eine fragliche radiologisch-pathologische Korrelation zu einer Narbe gegeben war. Die Anamnese ergab keinen Hinweis auf eine erfolgte Operation, ein Trauma oder eine Entzündung, sodass in der multidisziplinären Konferenz die Läsion als nicht geklärt eingestuft wurde.

Zur weiteren Abklärung des Dignitätsgrades wurde eine MR-Mammografie indiziert. Bei MR-mammografisch guter Beurteilbarkeit (minimale Bewegungsartefakte, MR-ACR 1) bestätigte sich ein irregulärer Herdbefund ( $\mathbf{A b b}$. 3 ) ohne suspekte Kontrastmitteldynamik als Korrelat der mammografischen und sonografischen Befunde. In Zusammenschau erschien trotz hoch suspekter Morphologie das Vorliegen eines invasiven Karzinoms unwahrscheinlicher als primär angenommen; als Differenzialdiagnosen wurden eine radiäre Narbe oder ein fibrosiertes invasives Karzinom diskutiert. Aufgrund der Diskrepanz zur Histologie und Anamnese wurde die Indikation zur sonografischen Re-Stanzbiopsie (14 G) gestellt mit Gewinnung von fünf weiteren bildgebend repräsentativen Zylindern. Die Proben ergaben erneut vernarbtes Gewebe mit tumoröser Infiltration durch histiozytenartige Zellen. Die gezielte immunhistoche-

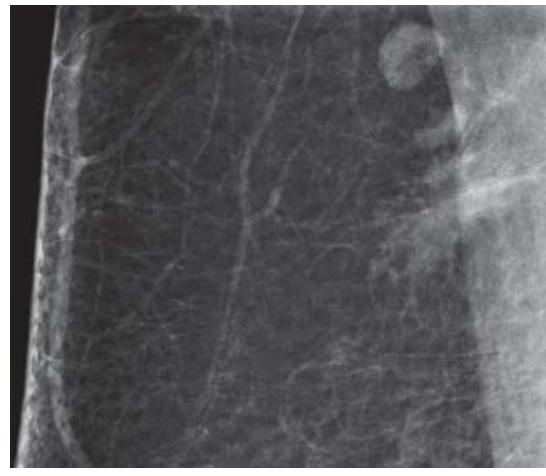

Abb. 1 Digitale Mammografie mit Kompression: An Lymphknoten angrenzender unscharfer bis gering spikulierter Herd.

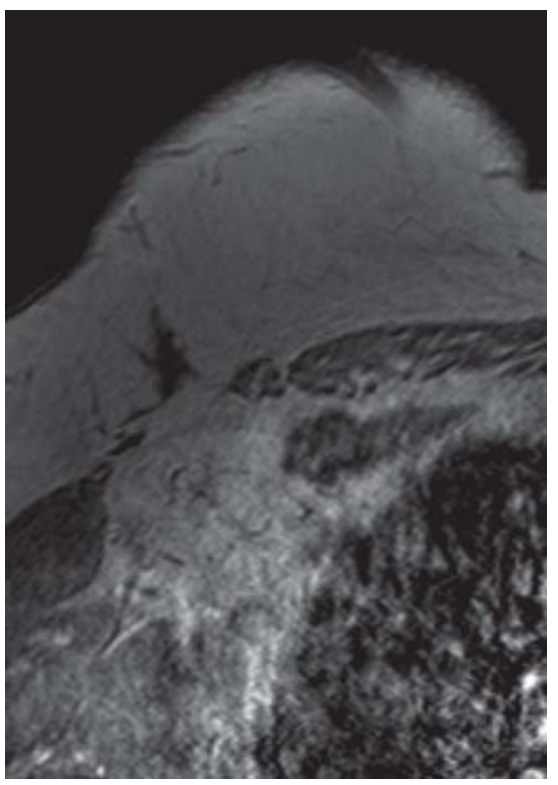

- Abb. 3 MR-Mammografie: Als Korrelat zur Sonografie und zur Mammografie präaxillärer, dreieckförmiger Herdbefund ohne suspekte Kontrastmitteldynamik.

mische Zusatzuntersuchung zeigte eine kräftige zytoplasmatische Expression von S100 ( Abb. 4), sodass in Zusammenschau sämtlicher Befunde die Diagnose eines Granularzelltumors gestellt werden konnte (unklares malignes Potenzial (B3), plausible radiologisch-pathologische Korrelation). Aufgrund eines typischen lokal progressiven Wachstums wurde eine Exzision im Gesunden angeraten. Maligne

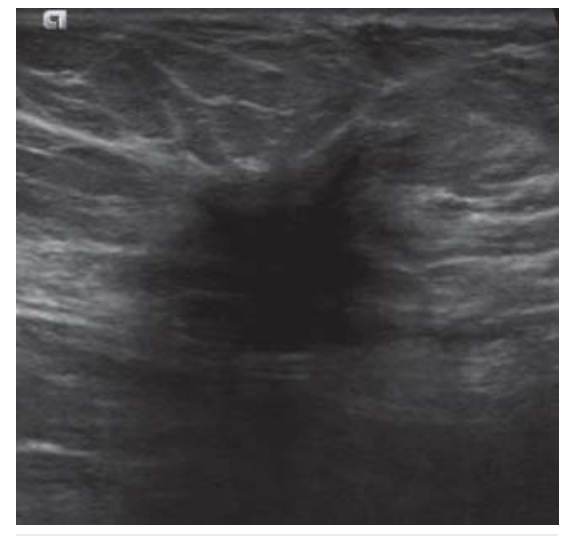

- Abb. 2 Sonografie: Echoarmer, unscharfer, gering spikulierter Herdbefund.

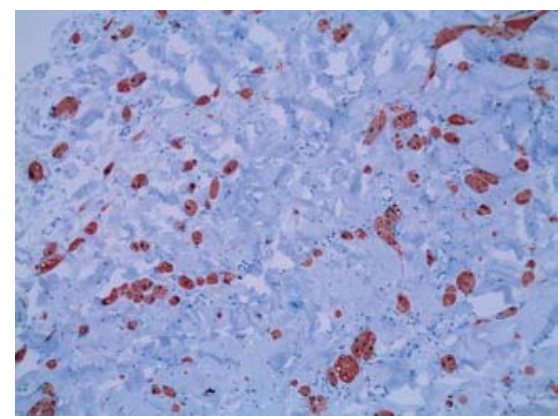

- Abb. 4 Immunhistochemie: Die Zellen des Granularzelltumors exprimieren typischerweise kräftig S100 (braun).

Verläufe sind rar, im Vordergrund steht die Rezidivprophylaxe.

\section{Diskussion}

Der 1926 erstmals von Abrikossoff fälschlicherweise myogenen Ursprungs beschriebene Granularzelltumor ist eine seltene, meist benigne Tumorentität, die in lediglich 5-6\% der Fälle in der Brust auftritt (Adeniran A et al. The Breast Journal 2004; 10: 528 - 531). Die Prävalenz im Vergleich zum Mammakarzinom wird mit 1 von 1000 angegeben (Brown AC et al. Surg Oncol 2011; 20: 97 -105). Zumeist sind prämenopausale Frauen mit einer angedeuteten Prädominanz von Frauen afrikanischer Herkunft betroffen. Die Genese ist unklar, wobei heutzutage 
davon ausgegangen wird, dass der Granularzelltumor entgegen Abrikossoffs Annahme eher neurogener Abstammung von Schwann-Zellen ist, intramammär häufig im inneren oberen Quadranten lokalisiert (Gavriilidis P et al. BM] Case Rep 2013; doi: 10.1136/bcr-2012-008178).

Da spezifische bildmorphologische Kriterien fehlen, ist die Diagnose insbesondere aufgrund der divergierenden Befundkonstellationen mit malignitätssuspekter Bildmorphologie und benigner Histologie oft protrahiert, wie im geschilderten Fall. Mammografisch ist der Granularzelltumor von glatt begrenzt bis spikuliert morphologisch indifferent und meist ohne Mikrokalkassoziation. Der Granularzelltumor präsentiert sich sonografisch häufig als hypoechogener Herd, ggf. in Assoziation mit einem dorsalen Schallschatten. MRmammografisch kann der Granularzelltumor stark Kontrastmittel anreichern und ist dann nicht von einem Karzinom zu unterscheiden. Allerdings entkräftet eine fehlende suspekte MR-tomografische Anreicherung einen spikulierten Herd, der anamnestisch keiner postoperativen Narbe zuzuordnen ist, nicht hinreichend. Daher ist der additive Einsatz der MRMammografie zur Vermeidung einer Re-
Stanzbiopsie kritisch zu diskutieren (Yau EJ et al. Breast J 2011; 17: 273 - 280).

Die Diagnose gelingt durch eine sorgfältig durchgeführte Diagnostik und den gezielten immunhistochemischen Nachweis einer starken Expression von Protein S100. Wäre die Diagnose eines Granularzelltumors bereits nach der ersten Biopsie interdisziplinär präsent gewesen, hätte durch den spezifischen Nachweis von S100 ggf. die korrekte Diagnose, die postoperativ bestätigt wurde, zu einem früheren Zeitpunkt gestellt werden können. Bei vereinzelt in der Literatur beschriebenen malignen Verläufen (Gupta $\mathrm{N}$ et al. Indian J Pathol Microbiol 2015; 58: 238 -240) und lokoregionären Rezidiven ist eine vollständige Exzision im Gesunden die Therapie der Wahl.

\section{Kernaussagen}

- Der Granularzelltumor der Brust ist ein meist benigner Tumor mit bildmorphologisch ggf. hoch suspekten Kriterien. Bei nicht konklusiver radiologischpathologischer Korrelation ist eine weitergehende Abklärung bis zur endgültigen Diagnosesicherung essenziell.
- Der Granularzelltumor ist eine Rarität und sollte, auch wenn nur wenige maligne Verläufe beschrieben sind, aufgrund seiner Rezidivwahrscheinlichkeit im Gesunden entfernt werden.

Korrespondenzadresse

\section{Dr. Philipp Polzer}

Institut für klinische Radiologie, Universitätsklinikum Münster

Albert-Schweitzer-Campus 1

48149 Münster

Germany

Tel.: ++49/1 76/61246243

Polzer.Philipp@googlemail.com

\section{Erstveröffentlichung}

DOI http://dx.doi.org/10.1055/s-0042108061

Fortschr Röntgenstr 2016; 188: 870-871

(c) Georg Thieme Verlag KG Stuttgart · New York · ISSN 1438-9029

\section{Bibliografie}

DOI http://dx.doi.org/10.1055/s-0043-105665 Senologie 2017; 14: 90-91

(c) Georg Thieme Verlag KG Stuttgart · New York ISSN 1611-6453 